\title{
Continuous constant pH Molecular Dynamics Simulations of Transmembrane Proteins
}

\author{
Yandong Huang ${ }^{1}$, Jack A. Henderson ${ }^{2 \star}$, and Jana Shen ${ }^{2}$ \\ ${ }^{1}$ College of Computer Engineering, Jimei University, Xiamen 361021, Fujian \\ Province, China. \\ ${ }^{2}$ University of Maryland School of Pharmacy, \\ Baltimore, MD 21201.
}

\begin{abstract}
Many membrane channels, transporters, and receptors utilize a $\mathrm{pH}$ gradient or proton coupling to drive functionally relevant conformational transitions. Conventional molecular dynamics simulations employ fixed protonation states, thus neglecting the coupling between protonation and conformational equilibria. Here we describe the membraneenabled hybrid-solvent continuous constant $\mathrm{pH}$ molecular dynamics method for capturing atomic details of proton-coupled conformational dynamics of transmembrane proteins. Example protocols from our recent application studies of proton channels and ion/substrate transporters are discussed.
\end{abstract}

Keywords: Molecular dynamics, electrostatics, $\mathrm{p} K_{a}$, Proton channels, Secondary active transporters, G-protein-coupled receptors

\section{Introduction}

\section{1 pH-dependent conformational dynamics of membrane proteins}

Solution pH plays a critical role in the functions of many transmembrane proteins, including secondary active transporters, proton channels, and G-proteincoupled receptors (GPCRs). For example, pH gradient across the membrane drives the transport of sodium ions through the sodium-proton antiporter NhaA, which is essential for cellular sodium and proton homeostasis in Escherichia coli $[54,60]$. Proton channels, such as the voltage-gated proton channel Hv1 [65], conduct protons across the membrane in response to a change in cellular $\mathrm{pH}[17,16]$. Solution $\mathrm{pH}$ also modulates the functions of membrane receptors. For example, the ovarian cancer GPCR1 and the closely related GPR4 regulate cell-mediated responses to acidosis in bone [51].

$\mathrm{pH}$-dependent transmembrane proteins often couple proton sensing with conformational changes [19]. For example, the inward- and outward-facing crystal structures of the sodium-proton antiporter NapA from Thermus thermophilus $[46,14]$ reveal an elevator-like alternating-access model, although the detailed mechanism of $\mathrm{pH}$-dependent conformational transition remains elusive. In many

\footnotetext{
* joint first author
} 
other cases, however, the conformational change is unknown due to the lack of active-state structure. For example, only inactive crystal structures have been resolved for the aforementioned antiporter NhaA $[37,47]$ and proton channel Hv1 [68]. Apart from high-resolution structure models, $\mathrm{p} K_{a}$ 's of active-site residues are important for mechanistic elucidation, as they inform protonation states at a certain $\mathrm{pH}$, thereby unveiling residues involved in proton binding/release coupled to the conformational transition. However, $\mathrm{p} K_{a}$ 's of membrane proteins are extremely challenging if possible at all to experimentally measure. Take NhaA as an example, electrophysiology and biochemical experiments established that it transports two protons in exchange for one sodium above $\mathrm{pH} 6.5[67,61]$; however, in the absence of an outward-facing crystal structure and experimental $\mathrm{p} K_{a}$ 's, the identities of proton-binding active-site residues remain controversial.

\subsection{Continuous constant $\mathrm{pH}$ molecular dynamics methods}

Constant $\mathrm{pH}$ molecular dynamics (pHMD) simulations $[9,72,12]$ offer a unique means to describe proton-coupled conformational dynamics and calculate $\mathrm{p} K_{a}$ values $[72,76,3]$. Current pHMD methods can be grouped into two classes. In discrete pHMD (DpHMD) methods [5,55], protonation states of titratable groups are periodically updated using Monte-Carlo sampling following molecular dynamics steps. Rooted in the $\lambda$-dynamics method for free energy calculations [45], continuous constant-pH molecular dynamics (CpHMD) methods [48, 41, $73,18,74,13,34,4,35,26,27]$ make use of fictitious $\lambda$ particles to sample protonation states simultaneously with atomic coordinates. A $\lambda$ particle carries a mass of a regular heavy atom (e.g., 10 atomic mass units) and the $\lambda$ coordinate continuously evolves between 0 and 1 , where 0 and 1 represent protonated and deprotonated states, respectively. The total Hamiltonian includes the kinetic energy of the fictitious particles and the nonbonded term is modified by linearly scaling electrostatic and van der Waals energies according to $\lambda$ coordinates. Thus, CpHMD directly couples conformational dynamics of the protein with protonation equilibria of all ionizable sites at a specified $\mathrm{pH}$. Following a CpHMD simulation at a single $\mathrm{pH}$ or a set of replica-exchange CpHMD simulations at multiple $\mathrm{pH}$ conditions, the trajectories of $\lambda$ coordinates are extracted, allowing the calculation of $\mathrm{p} K_{a}$ 's for all titratable sites.

The first two CpHMD methods [48, 41], implemented in CHARMM molecular dynamics package [6], make use of the generalized Born (GB) implicit-solvent models for both proton titration and conformational dynamics. Since then, several CpHMD methods have been developed and implemented in CHARMM. The hybrid-solvent CpHMD [73] was developed, which takes advantage of accurate description of conformational states in explicit solvent and rapid convergence of protonation state sampling in GB solvent. Additionally, the $\mathrm{pH}$ replica-exchange scheme [73] was developed to accelerate convergence of protonation and conformational state sampling. To overcome the limitations of implicit-solvent models, all-atom $\mathrm{CpHMD}^{\mathrm{MS} \lambda \mathrm{D}}$ with force switching was developed that uses explicit solvent to sample both titration and conformational dynamics [26,27]. To avoid 
the potential artifacts due to electrostatic cutoff and to further improve accuracy, particle-mesh Ewald (PME) all-atom CpHMD [34] was developed. CpHMD methods have also been implemented in the popular Amber [8] and GROMACS [64] packages, including GBNeck2-CpHMD [35, 28] in Amber and the all-atom CpHMD [18] in GROMACS [64]. Here we focus on the extensively validated [75, $22,11,33,79,23,29,30,69] \mathrm{pH}$ replica-exchange hybrid-solvent CpHMD method in CHARMM [73], which has been further developed to enable mechanistic studies of membrane proteins [33].

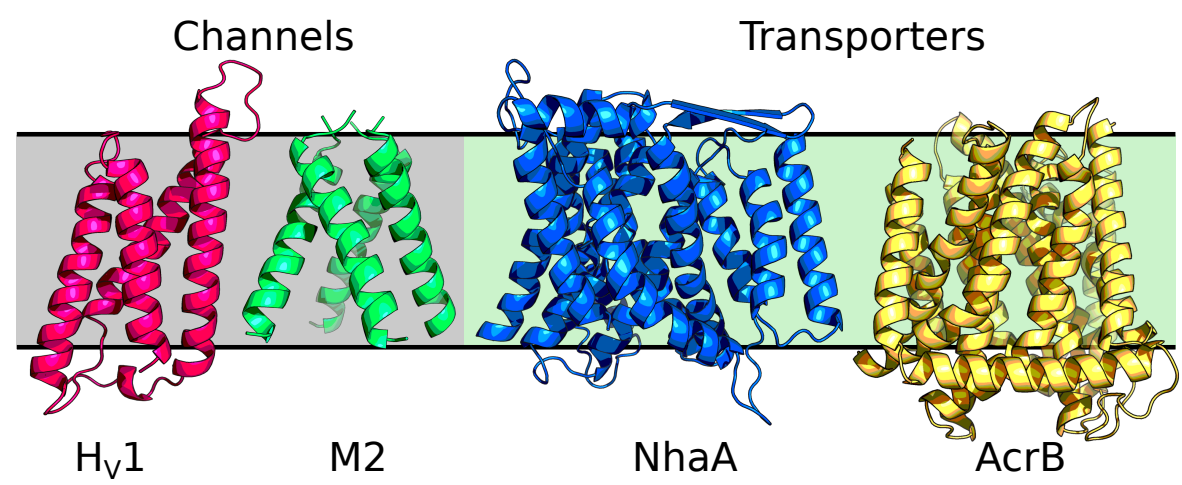

Fig. 1. Transmembrane proteins that have been studied by membrane-enabled hybridsolvent CpHMD simulations with $\mathrm{pH}$ replica-exchange [33]: M2 proton channel [11] (PDB: 3LBW [2]), voltage-gated Hv1 proton channel (Henderson and Shen, unpublished data, PDB: 3WKV [68]), sodium-proton antiporter NhaA[33] (PDB: 4AU5 [47]), and multi-drug efflux pump AcrB[79] (PDB: 4DX5 [21]).

\subsection{Membrane-enabled hybrid-solvent CpHMD with pH replica-exchange}

In the membrane-enabled hybrid-solvent CpHMD method [33], conformational dynamics of a transmembrane protein is sampled in explicit lipids and water, while protonation states are sampled using the membrane GBSW model [38]. Combined with the $\mathrm{pH}$ replica-exchange protocol [73], this method allows detailed description of proton-coupled conformational dynamics of transmembrane proteins (Fig. 1). For example, in the simulation study of NhaA [33], a mechanism emerged that explains the $\mathrm{pH}$-dependent activation of NhaA, reconciling a long standing controversy. According to the calculated $\mathrm{p} K_{a}$ values and conformational dynamics [33], Asp164 and Lys300 were identified as proton binding residues, consistent with the recent crystal structures of NhaA [47] and the homologous protein NapA [14]. When NhaA is exposed to the cytoplasmic pH, Asp164 releases the first proton and triggers the hydrophobic gate on the cytoplasm side to open, allowing the entrance of a sodium ion [33]. The sodium ion 
then binds to three active-site residues, which induces the release of a second proton from Lys300 [33]. The same protocol used to study NhaA has also been applied to investigate the proton-coupled conformational dynamics of the proton channel M2 from influenza virus [11] and the E. coli multi-drug efflux pump AcrB [79]. Most recently, a uniform electric field feature has been added to the membrane-enabled hybrid-solvent CpHMD simulations, allowing us to investigate the activation mechanism of a voltage-gated proton channel Hv1 (Fig. 2).

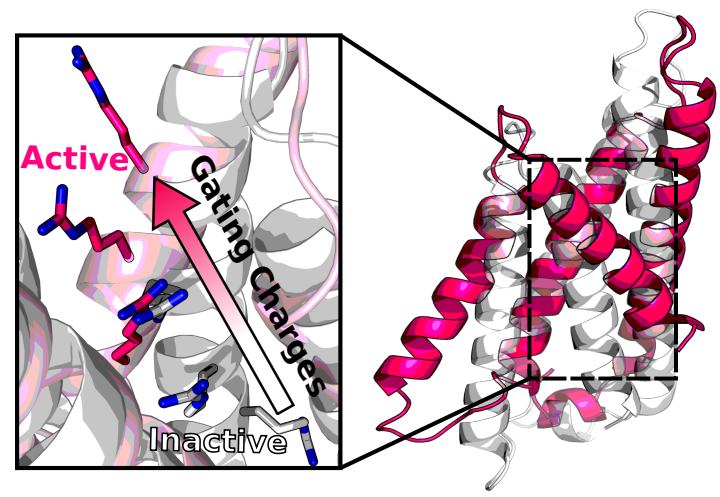

Fig. 2. Membrane-enabled hybrid-solvent CpHMD simulations with pH replicaexchange revealed conformational activation of the voltage-gated proton channel Hv1, as indicated by the upward movement of gating charges (Henderson and Shen, unpublished data).

Despite the recent successes of membrane-enabled hybrid-solvent CpHMD simulations (Fig. 1), several important limitations remain. For example, the implicit representation of solvent and bilayer employed in the titration dynamics limits the accuracy of $\mathrm{p} K_{a}$ estimation, particularly for buried active-site residues and those interacting with lipids or small-molecule ligands. In general, $\mathrm{p} K_{a}$ shifts relative to solution (model) $\mathrm{p} K_{a}$ are underestimated for interior residues and overestimated for lipid-interacting ones [33, 11,79]. Further, explicit interactions with lipids and ions, which may become important for protonation state changes, cannot be accounted for. While PME all-atom CpHMD [34] has the potential to overcome the limitations, our preliminary study of the M2 channel indicated that the calculated $\mathrm{p} K_{a}$ 's from all-atom $\mathrm{CpHMD}$ have larger systematic errors, likely a result of underpolarization with additive force fields. Thus, the hybridsolvent CpHMD method remains a method of choice until a solution is found to remedy the force field issue. A future direction is to incorporate polarizable force fields such as the Drude model [49] in PME all-atom CpHMD. Due to the high computational cost of simulations with polarizable force fields, a GPU implementation is desirable. 
bioRxiv preprint doi: https://doi.org/10.1101/2020.08.06.239772; this version posted August 6, 2020. The copyright holder for this preprint (which was not certified by peer review) is the author/funder, who has granted bioRxiv a license to display the preprint in perpetuity. It is made available under aCC-BY-ND 4.0 International license.

\section{Methods}

\subsection{Setting up a CpHMD simulation for transmembrane proteins}

Here we discuss the protocol of membrane-enabled hybrid-solvent CpHMD simulations in CHARMM [6], which can be divided into three stages (Fig. 3). In Stage 1, the protein/bilayer complex is constructed and undergoes an initial equilibration. In Stage 2, with the protein structure restrained the lipids are equilibrated to the appropriate structural and phase behavior. In Stage 3, protein sidechains are made titratable and CpHMD is invoked to equilibrate the system at a specified $\mathrm{pH}$ condition followed by production replica-exchange CpHMD at multiple $\mathrm{pH}$ conditions.

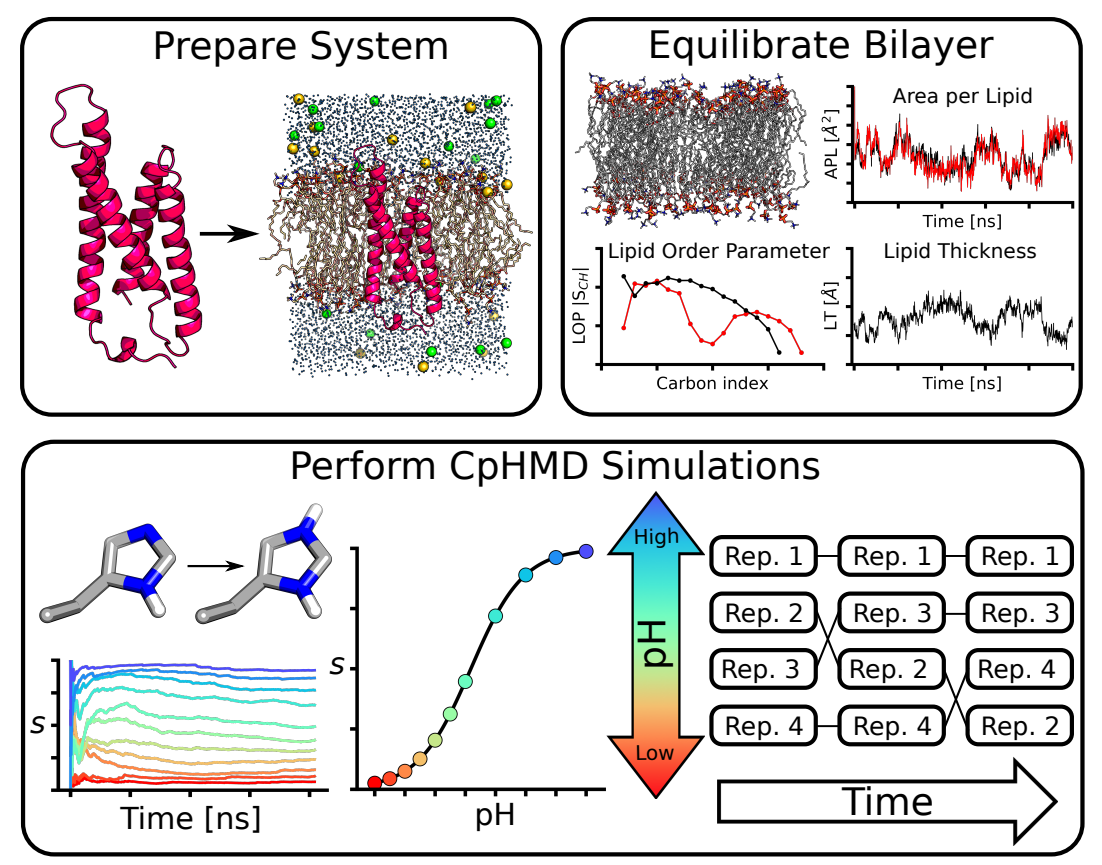

Fig. 3. Overview of the three-stage protocol for membrane-enabled hybrid-solvent CpHMD simulations with pH replica-exchange.

Stage 1. Prepare protein/bilayer complex. First, we retrieve the protein coordinates from the database, Orientations of Proteins in Membranes [50], which provides coordinates of membrane proteins by orienting the structure with respect to the membrane normal using theoretical calculations and comparisons to experimental data [50]. We then use MMTSB toolset [24] or other 
tools such as SWISS-MODEL [77] to add or replace missing residues, heavy atoms, or mutation sites. The HBUILD facility [7] in CHARMM is used to add missing hydrogens. Note, in this stage, all titratable sites are assumed to be in the "anticipated" protonation states based on the experimental $\mathrm{pH}$ condition and hydrogen bond/salt bridge patterns (for His). If no experimental $\mathrm{pH}$ can be found, default protonation states corresponding to physiological $\mathrm{pH}(7.4)$ should be used: $\operatorname{Asp}(-) / \operatorname{Glu}(-) / \operatorname{Lys}(+) / \operatorname{Arg}(+) / \operatorname{Cys}(0)$. Unless His is in a salt bridge, HSE or HSD (based on the hydrogen bonding pattern) should be used.

Since amino acid sequences given in a PDB file are often truncated, we suggest capping the terminal groups of each protein chain: for $\mathrm{N}$-terminus use $\mathrm{CH}_{3} \mathrm{CO}$ (patch $\mathrm{ACE}$ ), and for C-terminus use $\mathrm{NH}_{2}$ (patch CT2) or $\mathrm{NHCH}_{3}$ (patch CT3). However, if a terminal group interacts with a part of the protein, the free ionized form, $\mathrm{NH}_{3}^{+}$and $\mathrm{COO}^{-}$, should be used. Since CpHMD simulation will be conducted at multiple $\mathrm{pH}$ in Stage 3, we should consider possible protonation state changes. This consideration is especially relevant for $\mathrm{N}$-amino group, since its solution $\mathrm{p} K_{a}$ is 8.5 [59], only one unit above the physiological $\mathrm{pH}$. If N-amino group will be allowed to titrate, make sure the model parameters are included in the PHMD parameter file.

Once the protein structure is complete, a very short minimization in implicit solvent (e.g., with the GBSW model [39]) may be conducted with the steepest descent (SD) and adopted basis Newton-Raphson (ABNR) algorithms to relax unfavorable positions of hydrogens. It is important that during the minimization, protein heavy atoms should be fixed or restrained. For example, two rounds of 50 steps of SD followed by 10 steps of ABNR are performed, first with heavy atoms fixed and then with a harmonic force constant of $5 \mathrm{kcal} / \mathrm{mol} / \AA^{2}$. If an extensive number of atoms have been added we also suggest that these sites be energy minimized, but the overall structure should be kept as close as possible to the crystal structure. If crystal waters are resolved in the structure these should be retained.

The rest of Stage 1 follows the CHARMM-GUI protocol [40] for preparing a protein/membrane complex for MD simulations. The protein is placed in a lipid bilayer with water above and below the bilayer. Counterions for neutralizing the system and additional ions to reach the specified ionic strength are added. The assembled protein/membrane complex undergoes a series of initial equilibration steps (Table 1, Stage 1) to relax the lipid positions before subjecting to a lengthy membrane equilibration step in Stage 2.

Stage 2. Equilibrate bilayer. Once a protein/membrane complex is assembled and relaxed, the lipid bilayer needs to be fully equilibrated before protein conformational states can be sampled. Simulation in this stage can be carried out using any scalable simulation engine, such as GROMACS [1], NAMD [63], or OpenMM [20]. Typically, a 0.2-ns restrained MD is performed in the NVT ensemble followed by a 100-ns restrained MD in the NPT ensemble (see Table 1, Stage 2). During the equilibration, protein backbone and side chain heavy atoms are restrained with a harmonic force constant of $1.0 \mathrm{kcal} / \mathrm{mol} / \AA^{2}$. 
Table 1. Example equilibration steps in all three stages

\begin{tabular}{|c|c|c|c|c|c|c|c|}
\hline \multicolumn{3}{|c|}{$\begin{array}{c}\text { Step Ensemble Timestep } \\
(\mathrm{fs})\end{array}$} & \multirow{2}{*}{$\begin{array}{c}\text { Length } \\
(\mathrm{ns})\end{array}$} & \multicolumn{4}{|c|}{$\begin{array}{c}\text { Restraint force constant } \\
\left(\mathrm{kcal} / \mathrm{mol} / \AA^{2}\right)\end{array}$} \\
\hline \multicolumn{3}{|c|}{ Stage 1. Initial equilibration } & & $\mathrm{BB}^{a}$ & $\mathrm{SC}+\mathrm{XV}$ & Lipid $^{b}$ & $\mathrm{BWat}^{c}$ \\
\hline 1 & NVT & 1 & 0.05 & 10.0 & 5.0 & 2.5 & 2.5 \\
\hline 2 & NVT & 2 & 0.05 & 5.0 & 5.0 & 2.5 & 2.5 \\
\hline 3 & NPT & 2 & 0.1 & 5.0 & 5.0 & 1.0 & 1.0 \\
\hline 4 & NPT & 2 & 0.1 & 5.0 & 2.5 & 0.5 & 0.5 \\
\hline 5 & NPT & 2 & 0.1 & 2.5 & 2.5 & 0.1 & 0.1 \\
\hline 6 & NPT & 2 & 0.1 & 2.5 & 2.5 & 0.0 & 0.0 \\
\hline \multicolumn{8}{|c|}{ Stage 2. Membrane equilibration $\mathrm{BB}+\mathrm{SC}$} \\
\hline 1 & NVT & 2 & 0.2 & 2.5 & & & \\
\hline 2 & NPT & 2 & 100 & 2.5 & & & \\
\hline \multicolumn{4}{|c|}{ Stage 3. CpHMD equilibration } & $\mathrm{BB}+\mathrm{S}$ & & & \\
\hline 1 & NVT & 2 & 0.1 & 1.0 & & & \\
\hline 2 & NPT & 2 & 0.1 & 1.0 & & & \\
\hline 3 & NPT & 2 & 0.8 & 0.0 & & & \\
\hline
\end{tabular}

${ }^{a} \mathrm{BB}$ and $\mathrm{SC}$ refer to protein backbone and side chain heavy atoms, respectively. XWat and BWat refer to crystallographic and bulk water oxygen atoms, respectively. ${ }^{b}$ Lipid heavy atoms are restrained with a planar harmonic potential to ensure that lipid tails are close to the bilayer center $(-5 \AA<Z<5 \AA)$, and lipid head groups are close to the bilayer surface $\left(\mathrm{Z}= \pm 19 \AA\right.$ for POPE). ${ }^{c}$ A planar harmonic restraint is applied to the bulk water oxygen atoms to keep them away from the membrane hydrophobic region.

Surface area per lipid, bilayer thickness, and lipid order parameters are quantities commonly used to assess the phase behavior and fine structure of the simulated bilayer [43]. A lipid bilayer is considered equilibrated once all three quantities are converged to experimental values for the specified lipid type or those obtained in the force field validation study [43]. The program VTMC [56] can be used to calculate surface area per lipid; it excludes lipids within $5 \AA$ from the protein, as protein-lipid interactions can artificially decrease the value. Bilayer thickness here refers to the hydrophobic thickness, which is the distance between the average $Z$-positions of the lipid $\mathrm{C} 2$ atoms from the top and bottom leaflets. Lipid order parameters $S_{\mathrm{CH}}$ indicate the rigidity of the lipid tails [71] and are defined as

$$
S_{\mathrm{CH}}=\frac{1}{2}\left\langle 3 \cos ^{2} \theta-1\right\rangle,
$$

where $\theta$ is the angle between the $\mathrm{C}-\mathrm{H}$ bond of the lipid tail methylene and the membrane normal $(z)$, and the angular brackets denote ensemble average. $S_{\mathrm{CH}}$ can be calculated using a Tcl script for VMD [36].

Stage 3. Perform CpHMD titration simulations. Following equilibration of bilayer, the system can be further equilibrated using membrane-enabled [33] hybrid-solvent CpHMD simulation at a single $\mathrm{pH}$. From the final snap shot of Stage 2, dummy protons need to be added to all Asp, Glu, and His sidechains. 
For Asp and Glu, dummy protons are placed in the syn position. Note, the dihedral energy barrier to the rotation around the carboxylic bond is raised (to 3 $\mathrm{kcal} / \mathrm{mol}$ ) in the force field [41] to prevent a dummy hydrogen from moving to the less favorable anti position where it becomes unlikely to protonate ("ghost" proton) $[55,41]$. For His, a proton is added to either $\delta$ or $\epsilon$ nitrogen to form a doubly protonated HSP. Addition of dummy hydrogens requires a brief retrained energy minimization, e.g., two rounds of 50 steps of SD followed by 10 steps of ABNR. In the first round all heavy atoms are fixed in positions, and in the second round a harmonic restraint of $5.0 \mathrm{kcal} / \mathrm{mol} / \AA^{2}$ is applied to them. Next, three short restrained CpHMD simulations are performed at crystallization or physiological $\mathrm{pH}$ (see Table 1, Stage 3). The first and second CpHMD equilibration steps are run for $0.1 \mathrm{~ns}$ in the NVT and NPT ensembles, respectively, with protein heavy atoms restrained with a harmonic force constant of $1.0 \mathrm{kcal} / \mathrm{mol} / \AA^{2}$. The third unrestrained equilibration step is run in the NPT ensemble for $0.8 \mathrm{~ns}$. To prevent lateral drift of the protein, a cylindrical restraint with a harmonic force constant of $0.1 \mathrm{kcal} / \mathrm{mol} / \AA^{2}$ is applied using the MMFP facility in CHARMM [6].

Following equilibration at a single $\mathrm{pH}$, production CpHMD simulations can begin using the $\mathrm{pH}$ replica-exchange protocol [73]. First, a simulation $\mathrm{pH}$ range is selected by considering the active $\mathrm{pH}$ range of the protein or the one that represents experimental or physiological $\mathrm{pH}$ conditions. The $\mathrm{pH}$ replicas need to be placed close enough to obtain sufficient exchange probabilities (e.g., $>20 \%$ ), which requires overlap between the potential energy distributions of neighboring replicas. Typically, a $0.5 \mathrm{pH}$ unit increment provides desired exchange probabilities. Each replica is simulated in the NPT ensemble, with exchange attempts occurring every 500 or 1000 MD steps between adjacent replicas. We typically observe an average exchange rate of at least $40 \%$. More replicas at smaller intervals (e.g., $0.25 \mathrm{pH}$ units) may be added to increase the exchange rate. The effectiveness of sampling enhancement can be tracked by monitoring the movement of replicas through $\mathrm{pH}$ space as simulation proceeds. At least several replicas should move across the entire $\mathrm{pH}$ ladder to achieve enhanced sampling.

Settings in molecular dynamics. Protein is represented by CHARMM22/CMAP force field [52, 53], lipids and ions are represented by CHARMM36 force field [43], and water is represented by CHARMM modified TIP3P model. van der Waals energies are calculated using a switching function from 10 to $12 \AA$ with a cutoff at $14 \AA$, consistent with the protocol used in the development of CHARMM36 lipid force field [43]. Simulations are conducted under periodic boundary conditions with the PME method [15] for long-range electrostatic energies and forces. The real space calculation uses a $12-\AA \AA$ cutoff and the reciprocal space calculation uses a $1-\AA$ grid spacing and 6 th-order spline interpolation. The nonbond neighbor list is heuristically updated in CHARMM [6] or every $10 \mathrm{MD}$ steps in GROMACS [64].

To allow for a 2-fs integration time step, all bonds involving hydrogens are constrained using SHAKE [66] in CHARMM [6], LINCS [31] in GROMACS [64], or rigidBonds in NAMD [63]. In NPT simulations, temperature is maintained at 
$310 \mathrm{~K}$ by Nóse-Hoover thermostat $[58,32]$ and pressure is maintained at 1 atm by Langevin piston pressure-coupling algorithm in CHARMM [25] or ParrinelloRahman barostat [62] in GROMACS.

CpHMD specific settings. Unless otherwise noted, default CpHMD settings should be used. In membrane-enable hybrid-solvent CpHMD simulations [33] , the protein is centered in the lipid bilayer and an exclusion cylinder, e.g., with a radius of $25 \AA$, is placed along the membrane normal to exclude the low dielectric slab used in the implicit membrane model [38] from influencing the titration of interior residues in the protein. The cylinder should encompass the protein with no or minimal overlap with lipids. A center of mass restraint is applied to the protein with a harmonic force constant of $1.0 \mathrm{kcal} / \mathrm{mol} / \AA^{2}$ via the MMFP facility in CHARMM[6]. The thickness of the low-dielectric slab is set to the hydrophobic thickness of the lipid bilayer, defined as the difference between the average $z$ positions of lipid $\mathrm{C} 2$ atoms from the top and bottom leaflets, e.g., about $35 \AA$ for POPE. The half of membrane switching length [38] for the dielectric transition region between the low-dielectric slab and bulk water is set to $2.5 \AA$. The default GBSW atomic input radii $[57,10]$ are used for protein. For small molecules, the generic atomic radii in the GBSW radii file or van der Waals radii in the force field [44] should be used. Titration $(\lambda)$ and tautomeric state $(x)$ coordinates are updated every $5 \mathrm{MD}$ steps (default for hybrid-solvent CpHMD), allowing for water and lipid relaxation [73]. Langevin dynamics with a friction coefficient of $5 \mathrm{ps}^{-1}$ is used (default for CpHMD). The mass of $\lambda$ and $x$ particles are set to 10 atomic mass units (default for CpHMD).

\section{$2.2 \mathrm{p} K_{a}$ calculations}

To calculate the $\mathrm{p} K_{a}$ of a titratable site, the deprotonated fraction at each $\mathrm{pH}$, $S(\mathrm{pH})$, is obtained and fit to the generalized Henderson-Hasselbalch equation (Hill equation).

$$
S(\mathrm{pH})=\frac{1}{1+10^{n\left(\mathrm{pK}_{\mathrm{a}}-\mathrm{pH}\right)}},
$$

where $n$ is the Hill coefficient representing the slope of the transition region in the titration curve. Deviation of $n$ from 1 indicates the extent of cooperative $(n$ $>1)$ or anticooperative $(n<1)$ interactions between two or more titrating sites [72]. The deprotonated fraction of a titratable site is calculated as the fraction of time $\lambda$ samples the deprotonated state,

$$
S=\frac{N_{\text {deprot }}}{N_{\text {deprot }}+N_{\text {prot }}},
$$

where deprotonated and protonated states are defined as $\lambda>0.8$ and $\lambda<0.2$, respectively.

For coupled titrating sites, i.e. titration of one site is affected by the protonation state of the other, apparent or macroscopic sequential $\mathrm{p} K_{a} \mathrm{~s}$ are of interest [28]. In the statistical mechanics formulation, the two sequential $\mathrm{p} K_{a}$ 's, $\mathrm{p} K_{1}$ and 
$\mathrm{p} K_{2}$, are obtained by fitting the total number of protons bound to the two sites, $N_{\text {prot }}$, to the coupled titration model below [70,74].

$$
N_{\text {prot }}=\frac{10^{\mathrm{p} K_{2}-\mathrm{pH}}+2 \times 10^{\mathrm{p} K_{1}+\mathrm{p} K_{2}-2 \mathrm{pH}}}{1+10^{\mathrm{p} K_{2}-\mathrm{pH}}+10^{\mathrm{p} K_{1}+\mathrm{p} K_{2}-2 \mathrm{pH}}}
$$

Alternatively, the total deprotonated fraction of the two sites can be fit to the following uncoupled model [78, 27],

$$
S_{1}+S_{2}=\frac{1}{1+10^{\mathrm{p} K_{1}-\mathrm{pH}}}+\frac{1}{1+10^{\mathrm{p} K_{2}-\mathrm{pH}}}
$$

where $S_{1}$ and $S_{2}$ are the deprotonated fractions of the two sites. $\mathrm{p} K_{1}$ and $\mathrm{p} K_{2}$ are the two uncoupled $\mathrm{p} K_{a}$ 's.

\section{Acknowledgement}

The authors acknowledge National Institutes of Health (R01GM098818 and R01GM118772) for funding.

\section{References}

1. Abraham, MJ, Murtola, T, Schulz, R, Páll, S, Smith, JC, Hess, B, Lindahl, E (2015) GROMACS: High performance molecular simulations through multi-level parallelism from laptops to supercomputers. SoftwareX 1-2, 19-25

2. Acharya, R, Carnevale, V, Fiorin, G, Levine, BG, Polishchuk, AL, Balannik, V, Samish, I, Lamb, RA, Pinto, LH, DeGrado, WF, Klein, ML (2010) Structure and mechanism of proton transport through the transmembrane tetrameric M2 protein bundle of the influenza A virus. Proc. Natl. Acad. Sci. USA 107(34), 15075-15080

3. Alexov, E, Mehler, EL, Baker, N, Baptista, AM, Huang, Y, Milletti, F, Nielsen, JE, Farrell, D., Carstensen, T, Olsson, MHM, Shen, JK, Warwicker, J, Williams, $\mathrm{S}$, Word, JM (2011) Progress in the prediction of $\mathrm{p} K_{a}$ values in proteins. Proteins $79,3260-3275$

4. Arthur, EJ, Brooks, III, CL (2016) Efficient implementation of constant pH molecular dynamics on modern graphics processors. J. Comput. Chem. 37, 2171-2180

5. Baptista, AM, Teixeira, VH, Soares, CM (2002) Constant-pH molecular dynamics using stochastic titration. J. Chem. Phys. 117, 4184-4200

6. Brooks, BR, Brooks, III, CL, Mackerell, Jr, AD, Nilsson, L, Petrella, RJ, Roux, B, Won, Y, Archontis, G, Bartels, C, Boresch, S, Caflisch, A, Caves, L, Cui, Q, Dinner, AR, Feig, M, Fischer, S, Gao, J, Hodoscek, M, Im, W, Kuczera, K, Lazaridis, T, Ma, J, Ovchinnikov, V, Paci, E, Pastor, RW, Post, CB, Pu, JZ, Schaefer, M, Tidor, B, Venable, RM, Woodcock, HL, Wu, X, Yang, W, York, DM, Karplus, M (2009) CHARMM: the biomolecular simulation program. J. Comput. Chem. 30, 15451614

7. Brünger, AT, Karplus, M (1988) Polar hydrogen positions in proteins: Empirical energy placement and neutron diffraction comparison. Proteins 4, 148-156 
8. Case, DA, Ben-Shalom, IY, Brozell, SR, Cerutti, DS, Cheatham, III, T, Cruzeiro, VWD, Darden, TA, Duke, RE, Ghoreishi, D, Gilson, MK, Gohlke, H, Goetz, AW, Greene, D, Harris, RC, Homeyer, N., Huang, Y, Izadi, S, Kovalenko, A, Kurtzman, T., Lee, TS, LeGrand, S, Li, P, Lin, C, Liu, J, Luchko, T, Luo, R., Mermelstein, DJ, Merz, KM, Miao, Y, Monard, G, Nguyen, C, Nguyen, H, Omelyan, I., Onufriev, A, Pan, F, Qi, R, Roe, DR, Roitberg, A, Sagui, C, Schott-Verdugo, S, Shen, J, Simmerling, CL, Smith, J, Salomon-Ferrer, R, Swails, J, Walker, RC, Wang, J, Wei, H, Wolf, RM, Wu, X, Xiao, L, York, DM, Kollman, PA (2018) AMBER 2018. San Francisco

9. Chen, J, Brooks III, CL, Khandogin, J (2008) Recent advances in implicit solventbased methods for biomolecular simulations. Curr. Opin. Struct. Biol. 18, 140-148

10. Chen, J, Im, W, Brooks, III, CL (2006) Balancing solvation and intramolecular interactions: toward a consistent generalized Born force field. J. Am. Chem. Soc. $128,3728-3736$

11. Chen, W, Huang, Y, Shen, J (2016) Conformational activation of a transmembrane proton channel from constant $\mathrm{pH}$ molecular dynamics. J. Phys. Chem. Lett. 7, 3961-3966

12. Chen, W, Morrow, BH, Shi, C, Shen, JK (2014) Recent development and application of constant $\mathrm{pH}$ molecular dynamics. Mol. Simul. 40, 830-838

13. Chen, W, Wallace, J, Yue, Z, Shen, J (2013) Introducing titratable water to allatom molecular dynamics at constant pH. Biophys. J. 105, L15-L17

14. Coincon, M, Uzdavinys, P, Nji, E, Dotson, DL, Winkelmann, I, Abdul-Hussein, S, Cameron, A.D., Beckstein, O, Drew, D (2016) Crystal structures reveal the molecular basis of ion translocation in sodium/proton antiporters. Nat. Struct. Mol. Biol. 23, 248-255

15. Darden, T, York, DM, Pedersen, L (1993) Particle mesh Ewald: An N $\log (\mathrm{N})$ method for Ewald sums in large systems. J. Chem. Phys. 98, 10089-10092

16. DeCoursey, TE (2018) Voltage and $\mathrm{pH}$ sensing by the voltage-gated proton channel, Hv1. J. R. Soc. Interface 15, 20180108

17. DeCoursey, TE, Hosler, J (2014) Philosophy of voltage-gated proton channels. J. R. Soc. Interface 11, 20130799

18. Donnini, S, Tegeler, F, Groenhof, G, Grubmüller, H (2011) Constant pH molecular dynamics in explicit solvent with $\lambda$-dynamics. J. Chem. Theory Comput. 7, 19621978

19. Drew, D, Boudker, O (2016) Shared molecular mechanisms of membrane transporters. Annu. Rev. Biochem. 85, 543-572

20. Eastman, P, Swails, J, Chodera, JD, McGibbon, RT, Zhao, Y, Beauchamp, KA, Wang, LP, Simmonett, AC, Harrigan, MP, Stern, CD, Wiewiora, RP, Brooks, BR, Pande, VS (2017) OpenMM 7: Rapid development of high performance algorithms for molecular dynamics. PLoS Comput. Biol. 13, e1005659

21. Eicher, T, Cha, H, Seeger, MA, Brandstätter, L, El-Delik, J, Bohnert, JA, Kern, WV, Verrey, F, Grütter, MG, Diederichs, K, Pos, KM (2012) Transport of drugs by the multidrug transporter AcrB involves an access and a deep binding pocket that are separated by a switch-loop. Proc. Natl. Acad. Sci. USA 109, 5687-5692

22. Ellis, CR, Shen, J (2015) pH-dependent population shift regulates BACE1 activity and inhibition. J. Am. Chem. Soc. 137, 9543-9546

23. Ellis, CR, Tsai, CC, Hou, X, Shen, J (2016) Constant pH molecular dynamics reveals pH-modulated binding of two small-molecule BACE1 inhibitors. J. Phys. Chem. Lett. 7, 944-949 
24. Feig, M, Karanicolas, J, Brooks III, CL (2004) MMTSB tool set: enhanced sampling and multiscale modeling methods for applications in structural biology. J. Mol. Graph. Model. 22, 377-395

25. Feller, SE, Zhang, Y, Pastor, RW, Brooks, BR (1995) Constant pressure molecular dynamics simulation: The Langevin piston method. J. Chem. Phys. 103, 4613-4621

26. Goh, GB, Knight, JL, Brooks III, CL (2012) Constant pH molecular dynamics simulations of nucleic acids in explicit solvent. J. Chem. Theory Comput. 8, 36-46

27. Goh, GB, Hulbert, BS, Zhou, H, Brooks, III, CL (2014) Constant pH molecular dynamics of proteins in explicit solvent with proton tautomerism. Proteins 82, $1319-1331$

28. Harris, RC, Shen, J (2019) GPU-Accelerated implementation of continuous constant $\mathrm{pH}$ molecular dynamics in Amber: $\mathrm{p} K_{a}$ predictions with single- $\mathrm{pH}$ simulations. J. Chem. Inf. Model. 59, 4821-4832

29. Harris, RC, Tsai, CC, Ellis, CR, Shen, J (2017) Proton-coupled conformational allostery modulates the inhibitor selectivity for $\beta$-secretase. J. Phys. Chem. Lett. 8, 4832-4837

30. Henderson, JA, Harris, RC, Tsai, CC, Shen, J (2018) How ligand protonation state controls water in protein-ligand binding. J. Phys. Chem. Lett. 9, 5440-5444

31. Hess, B, Bekker, H, Berendsen, HJC, Fraaije, JGEM (1997) LINCS: A linear constraint solver for molecular simulations. J. Comput. Chem. 18, 1463-1472

32. Hoover, WG (1985) Canonical dynamics: Equilibration phase-space distributions. Phys. Rev. A 31(3), 1695-1697

33. Huang, Y, Chen, W, Dotson, DL, Beckstein, O, Shen, J (2016) Mechanism of pHdependent activation of the sodium-proton antiporter NhaA. Nat. Commun. 7, 12940

34. Huang, Y, Chen, W, Wallace, JA, Shen, J (2016) All-Atom Continuous Constant pH Molecular Dynamics With Particle Mesh Ewald and Titratable Water. J. Chem. Theory Comput. 12, 5411-5421

35. Huang, Y, Harris, RC, Shen, J (2018) Generalized Born Based Continuous Constant pH Molecular Dynamics in Amber: Implementation, Benchmarking and Analysis. J. Chem. Inf. Model. 58, 1372-1383

36. Humphrey, W, Dalke, A, Schulten, K (1996) VMD: visual molecular dynamics. J. Mol. Graphics 14, 33-38

37. Hunte, C, Screpanti, E, Venturi, M, Rimon, A, Padan, E, Michel, H (2005) Structure of a $\mathrm{Na}^{+} / \mathrm{H}^{+}$antiporter and insights into mechanism of action and regulation by $\mathrm{pH}$. Nature $435,1197-1202$

38. Im, W, Feig, M, Brooks III, CL (2003) An implicit membrane generalized Born theory for the study of structure, stability, and interactions of membrane proteins. Biophys. J. 85, 2900-2918

39. Im, W, Lee, MS, Brooks, III, CL (2003) Generalized Born model with a simple smoothing function. J. Comput. Chem. 24, 1691-1702

40. Jo, S, Kim, T, Im, W (2007) Automated builder and database of protein/membrane complexes for molecular dynamics simulations. PLoS ONE 2, e880

41. Khandogin, J, Brooks, III, CL (2005) Constant pH molecular dynamics with proton tautomerism. Biophys. J. 89, 141-157

42. Khandogin, J, Brooks, III, CL (2006) Toward the accurate first-principles prediction of ionization equilibria in proteins. Biochemistry 45, 9363-9373

43. Klauda, JB, Venable, RM, Freites, JA, O'Connor, J.W., Tobias, DJ, MondragonRamirez, C, Vorobyov, I, MacKerell, Jr, AD, Pastor, RW (2010) Update of the CHARMM all-atom additive force field for lipids: validation on six lipid types. J. Phys. Chem. B 114, 7830-7843 
44. Knight, JL, Brooks, III, CL (2011) Surveying implicit solvent models for estimating small molecule absolute hydration free energies. J. Comput. Chem. 32, 2909-2923

45. Kong, X, Brooks, III, CL (1996) $\lambda$-dynamics: A new approach to free energy calculations. J. Chem. Phys. 105, 2414-2423

46. Lee, C, Kang, HJ, von Ballmoons, C, Newstead, S, Uzdavinys, P, Dotson, DL, Iwata, S, Beckstein, O, Cameron, AD, Drew, D. (2013) A two-domain elevator mechanism for sodium/proton antiport. Nature 501, 573-577

47. Lee, C, Yashiro, S, Dotson, DL, Uzdavinys, P, Lwata, S, Sansom, MSP, von Ballmoos, C, Beckstein, O, Drew, D, Cameron, AD (2014) Crystal structure of the sodium-proton antiporter NhaA dimer and new mechanistic insights. J. Gen. Physiol. $144,529-544$

48. Lee, MS, Salsbury, Jr, FR, Brooks, III, CL (2004) Constant-pH molecular dynamics using continuous titration coordinates. Proteins 56, 738-752

49. Lemkul, JA, Huang, J, Roux, B, MacKerell Jr, AD (2016) An empirical polarizable force field based on the classical Drude oscillator model: development history and recent applications. Chem. Rev. 116, 4983-5013

50. Lomize, M.A., Lomize, A.L., Pogozheva, I.D., Mosberg, H.I. (2006) OPM: orientations of proteins in membranes database. Bioinformatics 22, 623-625

51. Ludwig, MG, Vanek, M, Guerini, D, Gasser, JA, Jones, CE, Junker, U, Hofstetter, H, Wolf, RM, Seuwen, K (2003) Proton-sensing G-protein-coupled receptors. Nature 425, 93-98

52. MacKerell, Jr, AD, Bashford, D, Bellott, M, Dunbrack, Jr, RL, Evanseck, JD, Field, MJ, Fischer, S, Gao, J, Guo, H, Ha, S, Joseph-McCarthy, D, Kuchnir, L., Kuczera, K, Lau, FTK, Mattos, C, Michnick, S, Ngo, T., Nguyen, DT, Prodhom, B., Reiher, III, WE, Roux, B, Schlenkrich, M, Smith, JC, Stote, R, Straub, J., Watanabe, M, Wiórkiewicz-Kuczera, J, Yin, D, Karplus, M (1998) All-atom empirical potential for molecular modeling and dynamics studies of proteins. J. Phys. Chem. B 102, 3586-3616

53. MacKerell, Jr., AD, Feig, M, Brooks, III, CL (2004) Extending the treatment of backbone energetics in protein force fields: limitations of gas-phase quantum mechanics in reproducing protein conformational distributions in molecular dynamics simulations. J. Comput. Chem. 25, 1400-1415

54. Mager, T, Rimon, A, Padan, E, Fendler, K (2011) Transport mechanism and pH regulation of the $\mathrm{Na}^{+} / \mathrm{H}^{+}$antiporter NhaA from Escherichia coli. J. Biol. Chem. 286, 23570-23581

55. Mongan, J, Case, DA, McCammon, JA (2004) Constant pH molecular dynamics in generalized Born implicit solvent. J. Comput. Chem. 25, 2038-2048

56. Mori, T, Ogushi, F, Sugita, Y (2012) Analysis of lipid surface area in proteinmembrane systems combining Voronoi tessellation and Monte-Carlo integration methods. J. Comput. Chem. 33, 286-293

57. Nina, M, Beglov, D, Roux, B (1997) Atomic radii for continuum electrostatics calculations based on molecular dynamics free energy simulations. J. Phys. Chem. B 101, 5239-5248

58. Nosé, S (1984) A molecular dynamics method for simulations in the canonical ensemble. Mol. Phys. 52, 255-268

59. Nozaki, Y, Tanford, C (1967) Examination of titration behavior. Methods Enzymol. 11, 715-734

60. Padan, E (2014) Functional and structural dynamics of NhaA, a prototype for $\mathrm{Na}^{+}$ and $\mathrm{H}^{+}$antiporters, which are responsible for $\mathrm{Na}^{+}$and $\mathrm{H}^{+}$homeostasis in cells . Biochim. Biophys. Acta 1837, 1047-1062 
61. Padan, E, Danieli, T, Keren, Y, Alkoby, D, Masrati, G, Haliloglu, T, Ben-Tal, N, Rimon, A (2015) NhaA antiporter functions using 10 helices, and an additional 2 contribute to assembly/stability. Proc. Natl. Acad. Sci. USA 112, E5575-E5582

62. Parrinello, M, Rahman, A (1981) Polymorphic transitions in single crystals: A new molecular dynamics method. J. Appl. Phys. 52, 7182-7190

63. Phillips, JC, Braun, R, Wang, W, Gumbart, J, Tajkhorshid, E, Villa, E, Chipot, C, Skeel, RD, Kalé, L, Schulten, K (2005) Scalable molecular dynamics with NAMD. J. Comput. Chem. 26, 1781-1802

64. Pronk, S, Páll, S, Schulz, R, Larsson, P, Bjelkmar, P, Apostolov, R, Shirts, MR, Smith, JC, Kasson, PM, van der Spoel, D, Hess, B, Lindahl, E (2013) GROMACS 4.5: a high-throughput and highly parallel open source molecular simulation toolkit. Bioinformatics 29, 845-854

65. Ramsey, IS, Moran, MM, Chong, JA, Clapham, DE (2006) A voltage-gated protonselective channel lacking the pore domain. Nature 440, 1213-1216

66. Ryckaert, JP, Ciccotti, G, Berendsen, HJC (1977) Numerical Integration of the cartesian equations of motion of a system with constraints: molecular dynamics of $n$-alkanes. J. Comput. Phys. 23, 327-341

67. Taglicht, D, Padan, E, Schuldiner, S (1991) Overproduction and purification of a functional $\mathrm{Na}^{+} / \mathrm{H}^{+}$antiporter coded by nhaA (ant) from Escherichia coli. J. Biol. Chem. 266, 11289-11294

68. Takeshita, K, Sakata, S, Yamashita, E, Fujiwara, Y, Kawanabe, A, Kurokawa, T, Okochi, Y, Matsuda, M, Narita, H, Okamura, Y, Nakagawa, A (2014) X-ray crystal structure of voltage-gated proton channel. Nat. Struct. Mol. Biol. 21, 352-357

69. Tsai, CC, Yue, Z, Shen, J (2019) How electrostatic coupling enables conformational plasticity in a tyrosine kinase. J. Am. Chem. Soc. 141, 15092-15101

70. Ullmann, GM (2003) Relations between protonation constants and titration curves in polyprotic acids: a critical view. J. Phys. Chem. B 107, 1263-1271

71. Vermeer, LS, de Groot, BL, Réat, V, Milon, A, Czaplicki, J (2007) Acyl chain order parameter profiles in phospholipid bilayers: computation from molecular dynamics simulations and comparison with ${ }^{2}$ H NMR experiments. Eur. Biophys. J. 36, 919 931

72. Wallace, JA, Shen, JK (2009) Predicting $\mathrm{p} K_{a}$ values with continuous constant $\mathrm{pH}$ molecular dynamics. Methods Enzymol. 466, 455-475

73. Wallace, JA, Shen, JK (2011) Continuous constant pH molecular dynamics in explicit solvent with pH-based replica exchange. J. Chem. Theory Comput. 7, 2617-2629

74. Wallace, JA, Shen, JK (2012) Charge-leveling and proper treatment of long-range electrostatics in all-atom molecular dynamics at constant pH. J. Chem. Phys. 137, 184105

75. Wallace, JA, Shen, JK (2012) Unraveling a trap-and-trigger mechanism in the pH-sensitive self-assembly of spider silk proteins. J. Phys. Chem. Lett. 3, 658-662

76. Wallace, JA, Wang, Y, Shi, C, Pastoor, KJ, Nguyen, BL, Xia, K, Shen, JK (2011) Toward accurate prediction of $\mathrm{p} K_{a}$ values for internal protein residues: the importance of conformational relaxation and desolvation energy. Proteins 79, 3364-3373

77. Waterhouse, A, Bertoni, M, Bienert, S, Studer, G, Tauriello, G, Gumienny, R, Heer, FT, de Beer, TA, Rempfer, C, Bordoli, L, Lepore, R, Schwede, T (2018) SWISSMODEL: homology modelling of protein structures and complexes. Nucleic Acids Res. 46, 296-303

78. Webb, H, Tynan-Connolly, BM, Lee, GM, Farrell, D, O’Meara, F, Søndergaard, CR, Teilum, K, Hewage, C, McIntosh, LP, Nielsen, JE (2011) Remeasuring HEWL 
$\mathrm{p} K_{a}$ values by NMR spectroscopy: methods, analysis, accuracy, and implications for theoretical $\mathrm{p} K_{a}$ calculations. Proteins 79, 685-702

79. Yue, Z, Chen, W, Zgurskaya, HI, Shen, J (2017) Constant pH molecular dynamics reveals how proton release drives the conformational transition of a transmembrane efflux pump. J. Chem. Theory Comput. 13, 6405-6414

\section{Checklist of Items to be Sent to Volume Editors}

Here is a checklist of everything the volume editor requires from you:

The final LATEX source files

A final PDF file

A copyright form, signed by one author on behalf of all of the authors of the paper.

A readme giving the name and email address of the corresponding author. 